\title{
Design of integrated radar and communication system based on MIMO-OFDM waveform
}

\author{
Yongjun Liu*, Guisheng Liao, Zhiwei Yang, and Jingwei Xu \\ National Laboratory of Radar Signal Processing, Xidian University, Xi' an 710071, China
}

\begin{abstract}
Orthogonal frequency division multiplexing (OFDM) waveform enables radar and communication functions simultaneously, which encounters low angle resolution and poor data rate for traditional single input single output (SISO) systems. To solve these problems, an integrated radar and communication system (IRCS) with multiple input multiple output (MIMO) OFDM waveform is proposed. The different limitations of radar and communication in designing such a system are investigated. Then, an optimization problem is devised to obtain suitable system parameters, including the number of subcarriers, subcarrier spacing, number of symbols, pulse repetition frequency (PRF) and length of cyclic prefix (CP). Finally, to satisfy the requirements of both radar and communication, the IRCS parameters are derived in three typical cases. Several numerical results are presented to illustrate the demands of radar and communication, inconsistent or consistent, for the IRCS parameters and the superiority of the proposed system.
\end{abstract}

Keywords: integrated radar and communication, orthogonal frequency division multiplexing (OFDM), multiple input multiple output (MIMO), system parameter design.

DOI: $10.21629 /$ JSEE.2017.04.06

\section{Introduction}

With the development of technique, the identical platform requires more and more equipment to command, communicate, reconnoiter, etc. Nevertheless, it will increase the system weight, size, energy consumption, and complexity of operation, deteriorate the electromagnetic interference among different devices, and degrade the system performance. To solve the problem, the advanced multifunction radio frequency concept (AMRFC) was proposed in [1]. As stated in [1], the integrated system employs a comprehensive design method to integrate radar, electronic warfare $(\mathrm{EW})$, and communication subsystems in an identical platform with the system resources shared. On the other hand, with the development of transportation system, many

Manuscript received May 17, 2016.

*Corresponding author.

This work was supported by the National Natural Science Foundation of China (61231017; 61671352). enterprises and companies are putting their efforts into the intelligent transportation system (ITS) [2,3]. And it is imperative for the ITS to sense the surrounding circumstance and transmit/receive information. Consequently, the radar and communication devices will be indispensable and their integration will promote the development of the ITS.

Currently, the communication frequency bands are gradually extending to microwave frequency band and several of them have been overlapped with radar operation frequency band. In addition, the radio frequency front-ends of communication and radar are similar and their signal processing is digitalized [4]. Hence, it is feasible for radar and communication to utilize the common transmit antennas, front-ends and digital signal processors. Now, the key issue is the design of integrated waveform which can be used by radar as well as employed by communication.

At present, there have been a multitude of researches on the design of integrated waveform. These works can be classified into two main categories. One is based on the multiplexing technique including space division multiplexing (SDM) [5], time division multiplexing (TDM) [6], frequency division multiplexing (FDM) [7], and code division multiplexing (CDM) [8]. However, such a class has the same drawback that radar and communication cannot operate in some domain, simultaneously. For instance, the radar and communication cannot operate at the same time slot for the methods based on the TDM technique. The other is based on waveform sharing and has two types. One is that the communication information is hidden in the conventional radar waveforms $[9,10]$, the other is that the communication waveforms generally employed are either slightly changed or not [11]. For the integration of radar and communication using the communication waveform, there are many literatures regarding the orthogonal frequency division multiplexing (OFDM) waveform [12 - 15].

For communication applications, the OFDM waveform has been widely used in the digital television broadcasting (DTB), digital audio broadcasting (DAB), digital video 
broadcasting (DVB), wireless local area network (WLAN), etc. And some standard communication protocols referring to OFDM have been established by the IEEE [16]. That is owing to its merits of high frequency spectrum efficiency, flexible multi-carrier modulation, and easy synchronization and equalization. For radar applications, OFDM as radar waveform, that is OFDM radar, has been studied by many researchers. In [17], the authors used the OFDM with cyclic prefix (CP) to solve the Doppler ambiguities. In [18], the sparsity theory was employed to estimate the velocities and ranges of targets with OFDM radar. What is more, there have been several works with respect to space time adaptive processing (STAP) [19], synthetic aperture radar (SAR) imaging [20], target detection [21], etc. We can see that the OFDM is not only widely applied in communication, but also a suitable kind of radar waveform. It is intuitive to exploit the OFDM as the integrated radar and communication waveform.

The conventional OFDM waveform in communication is continuous in general. In contrast, it is noncontinuous for pulse radar. Moreover, most of the employed OFDM waveforms in radar consist of OFDM pulse train without CP which is indispensable for communication, and one pulse only contains one OFDM symbol. If the integrated radar and communication system (IRCS) employs the continuous OFDM waveform, the transmit and receive antennas require to be well separated, which is difficult to realize in practice, especially in those cases when either transmit or receive antennas are close to each other. If the integrated OFDM waveform is impulse, the transmit and receive antennas can be shared and the number of antennas will be reduced to half. Furthermore, the problem of isolation between transmit and receive antennas can be perfectly settled. For the communication applications, however, the data rate will decrease because the radar duty ratio is $10 \%$ in some cases, i.e., the operation time of communication becomes $10 \%$ of what it was. That is the cost of the integration of radar and communication using pulse OFDM waveform. On the other hand, the single input single output (SISO) system for radar has low resolution in space domain and its update rate of track is low, since it needs to rotate the antenna for wide area surveillance.

In order to deal with the aforementioned issues, it is a valid way to utilize the multiple input multiple output (MIMO) techniques both in radar and communication. Compared with the SISO system, the MIMO system in communication utilizes the space dimension of transmit antennas to improve throughput capability as well as signal-to-noise ratio (SNR) $[22,23]$. Furthermore, the MIMO techniques in radar can increase the size of virtual aperture to enhance the angle resolution and form multi- beams simultaneously to improve the update rate [24-26]. Hence, it is natural to combine the MIMO technique with OFDM waveform to perform the integration of radar and communication.

The MIMO-OFDM systems have been proposed in both radar and communication. In communication, the MIMOOFDM system transmits continuous OFDM waveform with $\mathrm{CP}$, and the transmitted waveforms at different antennas are not necessary to be orthogonal $[27,28]$. Whereas in radar, most of the MIMO-OFDM systems transmit pulse OFDM waveform with CP [29-32] or without CP [33,34], and each pulse contains only one OFDM symbol. Moreover, the transmitted waveforms are orthogonal. In [33] and [34], each antenna only transmits one subcarriers of the OFDM waveform. If these waveforms are employed in the IRCS, the data rate is low, even if each OFDM symbol is modulated by different information. In the IRCS, the MIMO-OFDM system in [35] transmits consecutive spectral-interleaved OFDM symbols with CP. However, it is not feasible for long range surveillance. The system in [36] transmits step-frequency sequence, and only several subcarriers are transmitted simultaneously. The data rate is low although it is applicable for long range surveillance.

In this paper, we propose a new system combining the MIMO technique with OFDM waveform to perform the integration of radar and communication. The proposed system can perform long range surveillance and has higher data rate and angle resolution. In the following sections, the proposed system will be discussed in detail. The detailed constraints and the design of integrated system parameters will be presented. In Section 2, the integrated radar and communication system combining MIMO technique with OFDM waveform is proposed. In Section 3, the limitations for radar and communication are discussed and an optimization problem is constructed to achieve suitable system parameters which satisfy the requirements of both applications. In Section 4, integrated system parameter design results for three typical cases are given and performance results are presented. Finally, conclusions are drawn and future research problems are given in Section 5.

\section{Integrated radar and communication system}

The transmitted waveform will influence the system structure, such as waveform generation, signal processing, etc. The transmitted waveform of proposed IRCS is on the basis of OFDM waveform and it can be utilized for both radar and communication. Whereas the OFDM waveforms for radar and communication, in general, are different in order to suffice their own demands. Their characteristics are discussed as follows. 


\subsection{Preliminaries of OFDM waveform}

\subsubsection{Preliminaries of OFDM waveform for SISO system}

The transmitted waveform of the conventional OFDM radar is depicted in Fig. 1(a). We observe that each transmitted pulse contains only one OFDM symbol without carrying the communication information. Even if it is modu- lated with information, not only is the communication data rate low, but also the synchronization is uneasy. Although the pulse repetition frequency (PRF) can be increased to improve the data rate, the maximum unambiguity range (MUR) will be decreased. This is due to the fact that the traditional OFDM radar waveform is devised without taking the communication requirements into consideration.

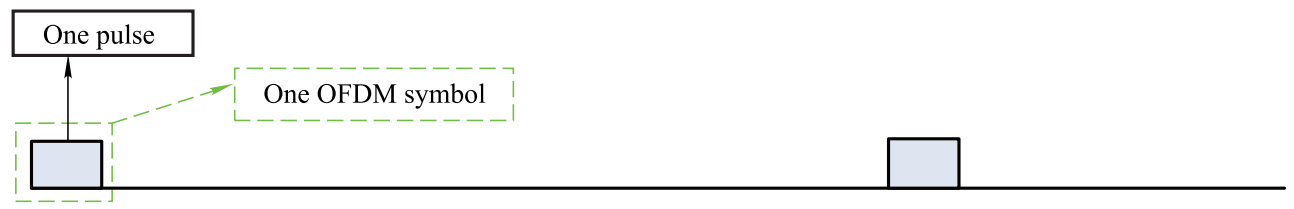

(a) Transmitted waveform of OFDM radar

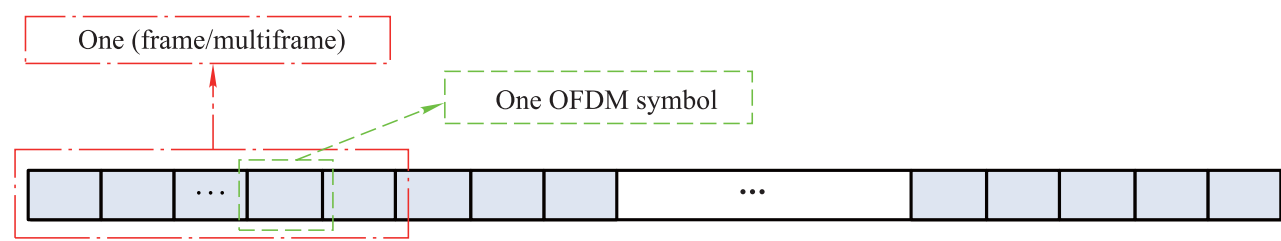

(b) Transmitted waveform of OFDM communication

Fig. 1 Transmitted waveform of OFDM radar and communication

The conventional OFDM communication waveform is shown in Fig. 1(b). The communication waveform consists of a series of OFDM symbols and is continuous. Compared with OFDM radar waveform, the continuity of waveform is their significant difference.

\subsubsection{Preliminaries of MIMO-OFDM waveform for communication}

The OFDM technique can transform frequency-selective fading channels into several independent flat fading subchannels, which the MIMO technique is incapable to do. However, the OFDM technique is sensitive to the Doppler shift, which will destroy the orthogonality among subcarriers. In contrast, the MIMO technique can solve the problem through space-time coding. In the MIMO-OFDM system for communication, the OFDM and MIMO techniques are combined to exert themselves advantages. To achieve both the coding an diversity gain, space-time code is utilized in the MIMO system, and several such systems combining the OFDM technique are proposed to solve inter-symbol interference (ISI), such as space-time trellis code OFDM (STTC-OFDM) [37], space-time block code OFDM (STBC-OFDM) [38,39], and space-frequency block code OFDM (SFBC-OFDM) [28,40]. In general, the waveforms of these systems can be summarized as in Fig. 2.

In Fig. 2, the transmitted signal of the $i$ th antenna is $s_{t, i}(t)$ with the subcarrier frequencies $f_{1}, f_{2}, \ldots, f_{N_{\mathrm{c}}}$. And all the antennas transmit continuous waveforms with the same subcarriers, but the subcarriers of different antennas are modulated by different space-time codes. The system throughput is increased through space diversity. However, the waveform is not suitable for the pulse radar with transmit and receive antennas shared.

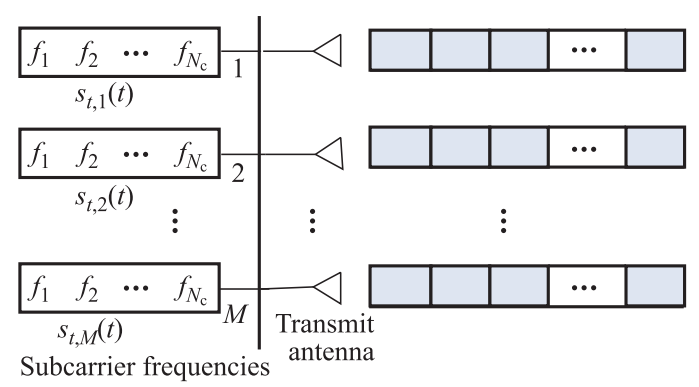

Fig. 2 MIMO-OFDM waveform for communication

\subsection{Integrated radar and communication system specifications}

\subsubsection{Integrated radar and communication for SISO system}

(i) Integrated waveform

To meet the IRCS's demands, the integrated waveform we proposed is depicted in Fig. 3. For the integrated waveform, several OFDM symbols are engaged in one pulse duration, and they constitute one frame or multiframe of 
communication to facilitate the communication synchronization. In general, by modulating each OFDM symbol with different information, we can improve the data rate and the pulse compression gain by at least $N_{s}$ times if one pulse contains $N_{s}$ OFDM symbols compared with the conventional OFDM radar waveform with the same bandwidth, subcarrier interval, PRF, and modulation scheme. However, the range of blind zone of radar will be enlarged, which is the price to be paid to improve the communication data rate. The ratio of the data rate of the integrated waveform to that of the conventional OFDM communication waveform in Fig. 1(b) is equal to the duty ratio of radar which is less than one. Therefore, the integrated waveform can be regarded as a tradeoff between OFDM radar and communication.

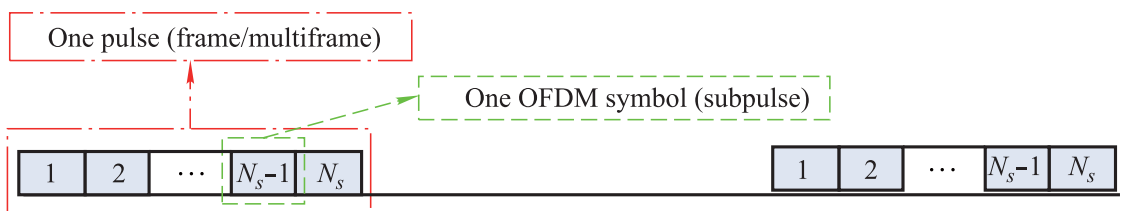

Fig. 3 Transmitted waveform of IRCS

(ii) Radar processing structure

The block diagram of transceiver architecture in IRCS is shown in Fig. 4. For simplicity, some structures are omitted. In the process of transmitting, direct digital synthesizer (DDS) including the communication information modula- tion generates the digital integrated baseband signal which is converted to the analog signal through digital to analog converter (DAC). And then it is up-converted to radio frequency (RF), amplified, and radiated through antenna.

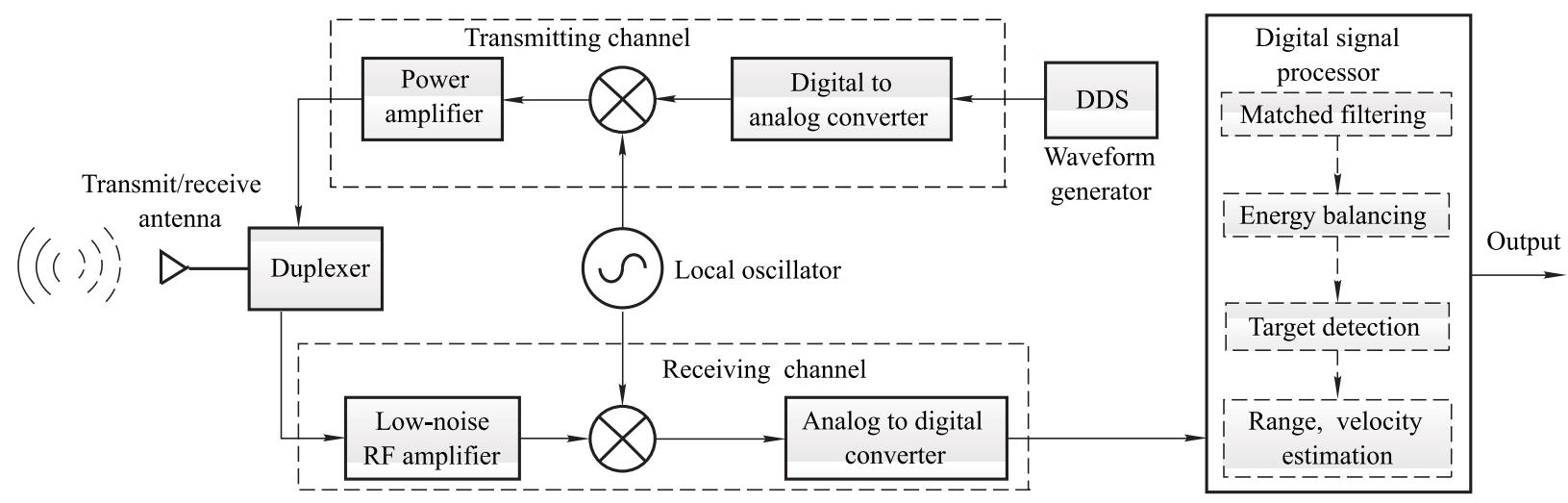

Fig. 4 Block diagram of transceiver architecture in IRCS

In the process of receiving, the echo is received and amplified by low-noise RF amplifier. And then the echo is down-converted to intermediate frequency (IF) band, baseband and then converted to digital signal by analog to digital converter (ADC). The digitalized baseband echoes are processed in the digital signal processor in which the matched filtering, i.e., pulse compression, is performed firstly. And then in order to compensate the different transmit energy due to the demand of communication, energy balancing for different pulses is performed. Afterwards, target detection is implemented and signal processing algorithms are employed to obtain the estimations of range and velocity or other results, for example the trajectory of moving targets. Eventually, the processing results are output to, for instance, display.

(iii) Communication processing structure

The block diagram of communication processing in the IRCS is illustrated in Fig. 5. The integrated waveform is so similar to the conventional OFDM waveform in communication that the processing in communication receiver is also similar. The integrated waveform is down-converted to baseband and converted to digital signal by ADC. Then the synchronization is performed, and the $\mathrm{CP}$ is removed. Finally, the data are output after fast Fourier transform (FFT) and decoding is executed. There is a slight difference from the conventional communication. When the synchroniza- 
tion is accomplished, the subsequent procedures are only performed during the pulse duration, that is to say, the processing is noncontinuous. As the time division multiple access (TDMA) for two users, the communication receiver can be regarded as a user and it only requires to demodulate the information during its time slot, i.e., the pulse duration.

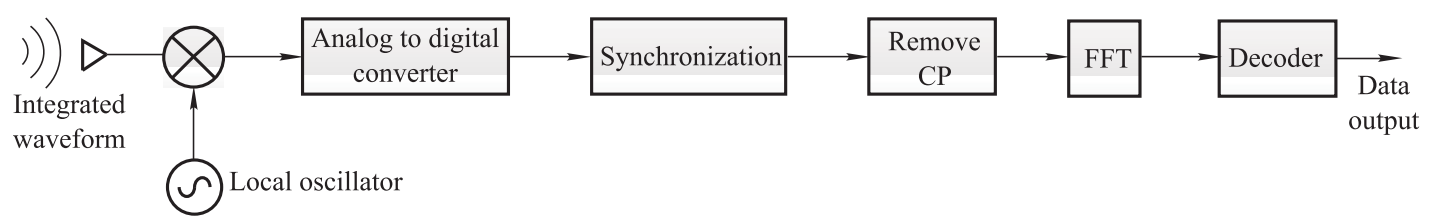

Fig. 5 Block diagram of processing for communication receiver

\subsubsection{MIMO-OFDM IRCS}

(i) Integrated waveform

The IRCS combining MIMO system with OFDM waveform is shown in Fig. 6, where the MIMO-OFDM IRCS consists of a linear equidistant antenna array with $M$ sensors and distance $d$. The allocated subcarrier frequencies at each antenna are depicted on the left side. The transmitted signal of the $i$ th antenna is $s_{t, i}(t)$ and the sub- carrier frequencies are $f_{i, 1}, f_{i, 2}, \ldots, f_{i, N_{c}}$, and $f_{i, m}=$ $f_{c}+\left[(i-1) N_{c}+m\right] \Delta f$ where $i$ indicates the element index; $m$ denotes the subcarrier frequency index; $f_{c}$ is the center frequency; $\Delta f$ is the subcarrier interval; and $N_{c}$ is the number of subcarriers engaged by each OFDM symbol. Compared with the MIMO-OFDM waveform for communication in Fig. 2, we can see that at each antenna, the subcarriers are same for MIMO-OFDM waveform whereas they are different for the integrated waveform of IRCS.

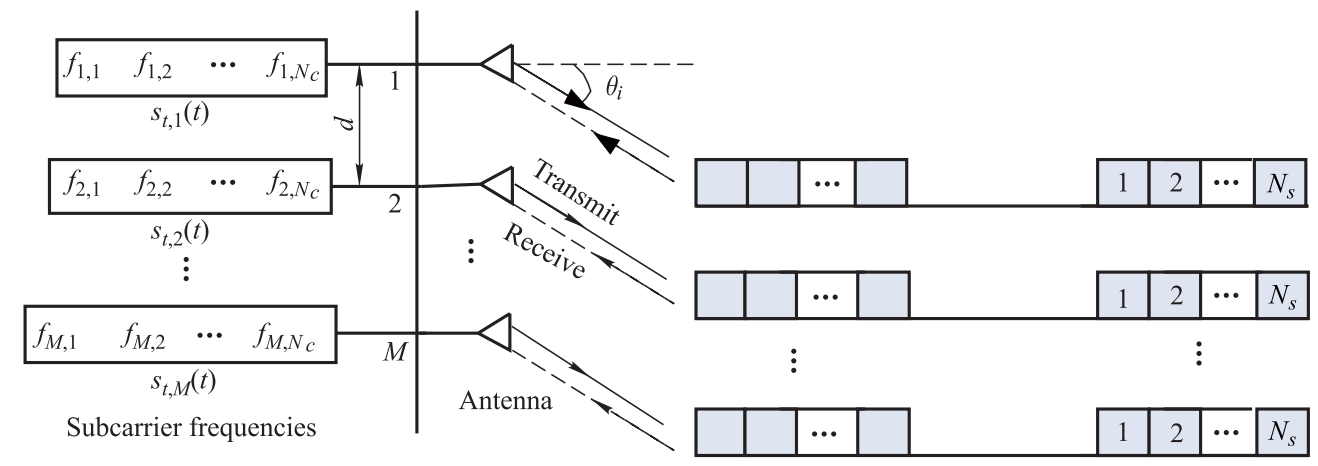

Fig. 6 Sketch map of MIMO-OFDM IRCS structure

Obviously, the integrated waveforms at different antennas are orthogonal to each other, which just satisfies the requirement of MIMO radar. For the IRCS, the size of virtual array aperture is two times larger than that of the real array aperture. In other words, the spatial resolution is improved by a factor of 2 . Furthermore, for communication, given a fixed bit error rate (BER), the data rate of the IRCS is improved compared with the SISO system, if each antenna transmits different information. And the data rate loss caused by the discontinuity of integrated waveform can be decreased or eliminated by MIMO technique. On the other hand, we can reduce the number of OFDM symbols of each pulse to lessen the range of the blind zone without more data rate loss compared with the SISO system.

(ii) Radar processing structure

The block diagram of MIMO-OFDM IRCS is depicted in Fig. 7. The transmitting and receiving channels are similar to that in Fig. 4. The slight difference is that the MIMOOFDM IRCS utilizes the identity DDS to generate the transmitted waveforms of all antennas, and in the DDS the communication information is modulated through spacetime coding. Moreover, all the transceivers utilize the same local oscillator. 


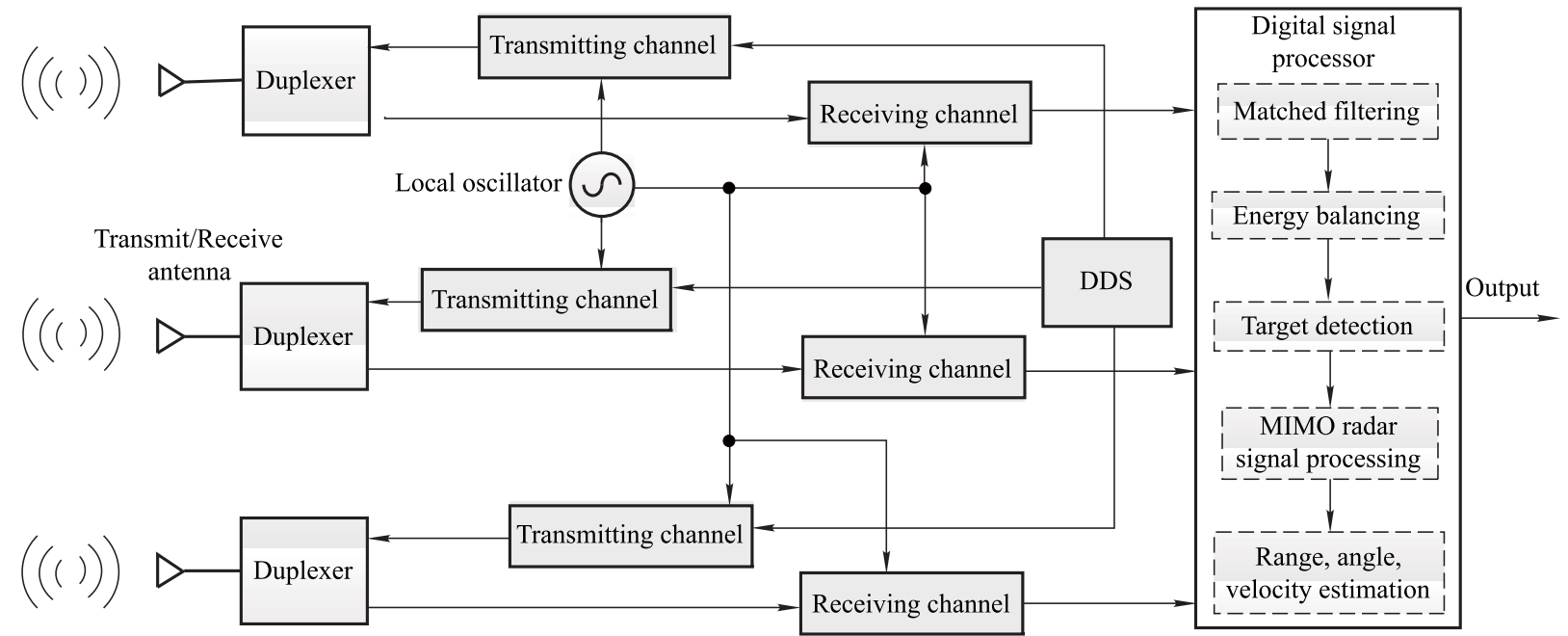

Fig. 7 Block diagram of MIMO-OFDM IRCS

All the digitalized outputs of receiving channels are processed in the digital signal processor in which the matched filtering i.e., pulse compression is carried out firstly, and then energy balancing and target detection are performed. Afterwards, some MIMO techniques and signal processing algorithms are exploited to obtain the estimations of range, angle and velocity, or other results. Finally, the processing results are output.

(iii) Communication processing structure
Fig. 8 shows the block diagram of communication processing in the MIMO-OFDM IRCS. Similar to the processing in Fig. 5, the FFT outputs of all channels are processed by space-time processing and decoding which is the inverse of the space-time coding in the transmitter. Similarly, the procedure of decoding is not continuous, either. And the available communication receiver is readily compatible with the IRCS.

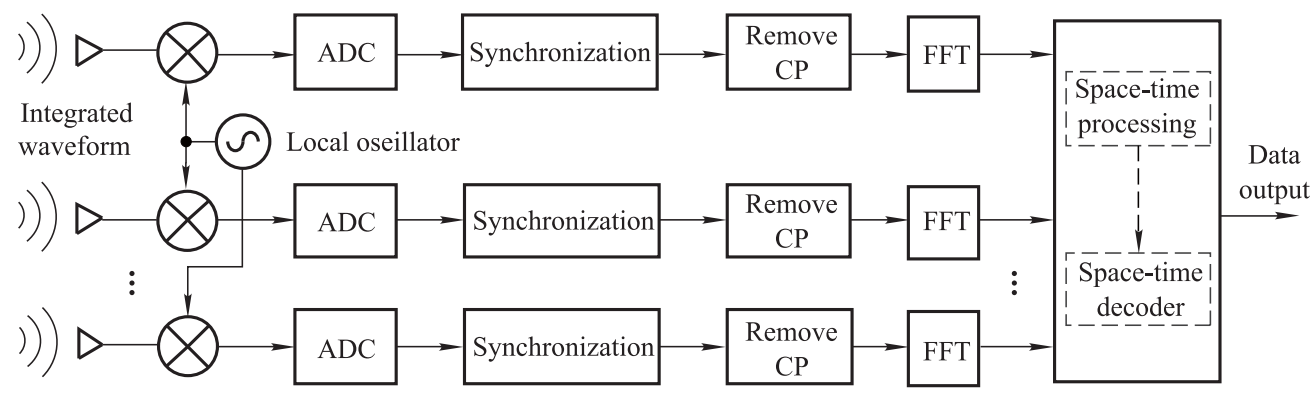

Fig. 8 Block diagram of processing for MIMO communication receiver

\section{System parameters design}

System parameters have a paramount influence on the system performance. For the IRCS, system parameters design should simultaneously meet the requirements of radar and communication. For radar system, the duty ratio $D$, PRF $f_{r}$, center frequency $f_{c}$, pulse duration $T_{p}$, signal bandwidth engaged by each antenna $B_{a}=N_{c} \Delta f$, system bandwidth $B_{s}=M N_{c} \Delta f$, and array element interspace $d$ are required to be devised. For communication, the integrated waveform consists of several OFDM symbols. Therefore, the parameters design coincides with the design of OFDM symbols.
The OFDM symbol is shown in Fig. 9. The length of CP or guard interval (GI) $T_{g}$, subcarrier frequency spacing $\Delta f=\frac{1}{T}$ with elementary symbol duration $T$, time duration of OFDM symbol $T_{s}$, signal bandwidth $B_{a}$, system bandwidth $B_{s}$, and duty ratio $D$ are the parameters associated with communication.

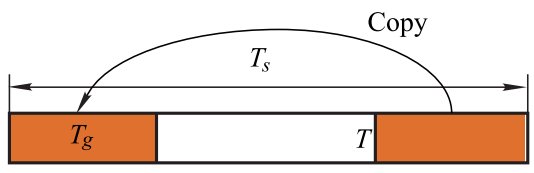

Fig. 9 OFDM symbol 


\subsection{Radar requirements}

For radar, the crucial performance indexes are Doppler tolerance, MUR, range resolution, maximum unambiguity velocity (MUV) and blind zone. In radar applications, the Doppler shift $f_{d r}$ is arisen from the radial velocity between IRCS and target

$$
f_{d r}=\frac{2 v_{r}}{\lambda}=\frac{2 v_{r} c_{0}}{f_{c}}
$$

where $v_{r}$ is the radial velocity between radar and target; $\lambda$ is the wavelength and $c_{0}$ is the speed of propagation. To keep the orthogonality of subcarriers, the maximum Doppler shift $f_{d r \text {, max }}$ must be far less than the subcarrier spacing $\Delta f$. In practice, it has been revealed that the subcarriers can be regarded as keeping orthogonal if $\Delta f \geqslant 10 f_{d r, \max }$.

The MUV $v_{u}$ and MUR $R_{u}$ of radar are associated with the PRF $f_{r}$. The following can be obtained:

$$
\begin{gathered}
v_{u}=\frac{c_{0} f_{r}}{2 f_{c}} \\
R_{u}=\frac{c_{0}}{2 f_{r}}=\frac{c_{0} T_{r}}{2}
\end{gathered}
$$

where $T_{r}=\frac{1}{f_{r}}$ is the pulse repetition interval (PRI).

For the IRCS in [35], the MUR $R_{I u}$ is

$$
R_{I u}=\frac{c_{0} T}{2 M} .
$$

Obviously, compared with the MUR in (3), $R_{I u}$ is much smaller than $R_{u}$, since $T_{r}>T$, and $M>1$. That is the reason why the IRCS in [35] is applicable for short range surveillance.

The radar blind zone $R_{b z}$ is given by

$$
R_{b z}=\frac{c_{0} T_{p}}{2}
$$

Evidently, the blind zone $R_{b z}$ is determined by the pulse duration $T_{p}=N_{s}\left(T_{g}+T\right)$. Another important performance parameter is the range resolution $\Delta R$ which is only determined by the entire signal bandwidth and has no relation to the signal shapes. The range resolution is as follows:

$$
\Delta R=\frac{c_{0}}{2 B_{s}}=\frac{c_{0}}{2 M N_{c} \Delta f} .
$$

We can see that the range resolution can be improved by increasing the entire signal bandwidth.

\subsection{Communication requirements}

For communication, the communication information is carried by OFDM signals. In the communication receiver, in order to correctly demodulate the information, the subcarriers of received signals are required to be orthogonal. However, the orthogonality will be destroyed by the Doppler shift $f_{d c}$ which is caused by the relative velocity between IRCS and the communication receiver. The Doppler shift can be achieved as follows:

$$
f_{d c}=\frac{v_{c}}{\lambda}=\frac{v_{c} c_{0}}{f_{c}}
$$

where $v_{c}$ indicates the radial velocity between IRCS and communication receiver. Compared with the expression in (1), the relative velocity in (7) is different from that in (1). Similarly, the maximum Doppler shift $f_{d c \text {, max }}$ requires to be much less than subcarrier spacing $\Delta f$. Identically, it is the same as the radar requirements that $\Delta f \geqslant 10 f_{d c \text {,max }}$. In addition, the ISI can also disrupt the orthogonality. To eliminate the ISI, the length of CP is no less than the maximum time delay $\tau_{\max }$ of communication channel, i.e.,

$$
T_{g} \geqslant \tau_{\max }
$$

The other requirement of communication is the channel capacity $C_{t}$. If there are $M$ receive antennas in communication receiver and the additive white Gaussian noise (AWGN) channels are orthogonal, the Shannon capacity can be written as

$$
C_{t}=D M B_{a} \log _{2}\left(1+\alpha \frac{P_{t}}{N_{0} B_{a}}\right)
$$

where $P_{t}$ indicates the transmit power; $N_{0}$ is the power spectral density of AWGN; and $\alpha$ is the propagation loss factor. In (9), the duty ratio $D=\frac{T_{p}}{T_{r}}$ is less than unity because the integrated waveform is noncontinuous. If $D=1$, (9) represents the AWGN Shannon capacity of continuous waveform in MIMO system with $M$ transmit and $M$ receive antennas. Hence, the duty ratio can be interpreted as the capacity loss factor due to the discontinuity of waveform.

\subsection{IRCS parameters design criterion}

The requirements of conventional radar and communication have been discussed in Section 3.1 and Section 3.2, respectively. It is seen that the radar and communication applications have some common requirements except for contradictions. For the IRCS, it is unavoidable to make a compromise in the system parameters due to the different purposes of radar and communication. To this end, we construct the following optimization problem as

$$
\begin{gathered}
{\left[\hat{N}_{c}, \hat{N}_{s}, \hat{T}_{g}, \hat{T}, \hat{f}_{r}\right]=} \\
\arg \max _{N_{c}, N_{s}, T_{g}, T, f_{r}} \frac{N_{s}\left(T_{g}+T\right) f_{r} M N_{c}}{T} \log _{2}\left(1+\frac{\alpha T P_{t}}{N_{0} N_{c}}\right)
\end{gathered}
$$




$$
\begin{gathered}
\text { s.t. } N_{s}\left(T_{g}+T\right) f_{r} \leqslant D^{0} \\
T_{g} \geqslant \tau_{\max } \\
1 / T \geqslant 10 \max \left\{f_{d r, \max }, f_{d c, \max }\right\} \\
N_{c} / T \leqslant B_{a}^{0} \\
\frac{c_{0}}{2 f_{r}} \geqslant R_{u}^{0} \\
\frac{c_{0}}{2} N_{s}\left(T_{g}+T\right) \leqslant R_{b z}^{0} \\
\frac{c_{0} T}{2 M N_{c}} \leqslant \Delta R^{0} \\
\frac{c_{0} f_{r}}{2 f_{c}} \geqslant v_{u}^{0}
\end{gathered}
$$

where $D^{0}, B_{a}^{0}, R_{u}^{0}, R_{b z}^{0}, \Delta R^{0}$ and $v_{u}^{0}$ are all user parameters corresponding to duty ratio, signal bandwidth, MUR, blind zone, range resolution and MUV thresholds, respectively. In (10), the center frequency $f_{c}$ and number of element $M$ are not included in the optimization variables, since $f_{c}$ and $M$ have a closely connection with practical application. Thus we assume that $f_{c}$ is at the frequency band occupied by both communication and radar, and both $f_{c}$ and $M$ have been given.

In the optimization problem (10), the constraint (10b) ensures the duty ratio to be no larger than the threshold $D^{0}$ to satisfy the application scenario, and the larger the duty ratio is, the greater the channel capacity will be. The constraint (10c) makes sure that the $\mathrm{CP}$ is no less than the maximum time delay of communication channel so that the ISI can be eliminated. The constraint (10d) guarantees that the orthogonality of subcarriers both for radar and communication functions is not destroyed by the Doppler shift. The constraint (10e) is the constraint on the signal bandwidth to satisfy the limit of hardware. In (10f) the MUR is no less than $R_{u}^{0}$ for the long range surveillance. The constraint $(10 \mathrm{~g})$ refines the bind zone to be no greater than $R_{b z}^{0}$. The constraint (10h) is to satisfy the demand for range identification. The requirement for MUV is guaranteed by the constraint (10i).

Obviously, the objective function and the inequality constraints (10b), (10e), (10g), and (10h) are nonconvex. Moreover, the inequality constraints (10c), (10d), (10f), (10i) are concave. Hence, the optimization problem in (10) is a multivariable nonconvex programming problem [41]. The analytical solution is unable to be obtained, but the numerical solution can be achieved by utilizing the evolutionary algorithms, such as the Genetic algorithm (GA) [42].

The optimization problem in (10) can be interpreted as that the object function is maximized to achieve the maximum channel capacity under the constraint conditions which guarantee that all the radar and partial communication requirements are satisfied. The solution to the optimization problem (10) can satisfy the IRCS requirements, since it gives consideration to both radar and communication limitations and concurrently maximizes the channel capacity. Consequently, the created optimization problem is reasonable.

\section{IRCS parameterization and simulation}

\subsection{IRCS parameterization}

From the previous discussion, the optimization problem in (10) is arduous to be solved. Generally, the conventional GA can be employed with the following parameters: the population size is 300 , the number of generation is 100 , the crossover probability is 0.9 , the mutation probability is 0.1 , and the roulette wheel selection is used. However, the object function can be interpreted as the function of duty ratio $D$ and signal bandwidth $B_{a}$. Analyzing the object function, we observe that it is monotonically increasing with $D$, and also monotonically increasing with $B_{a}$ if $\frac{\alpha P_{t}}{N_{0}}$ is large enough, which can be satisfied in most cases. So in the IRCS parameters design, we merely maximize $D$ and $B_{a}$ as much as possible according to practical applications without utilizing the complicated numerical algorithms. As an example, the IRCS parameterization procedure is given as follows.

In IEEE 802.11a, the radio frequencies of the local area network (LAN) system are aimed for the $5.15-5.25 \mathrm{GHz}$, $5.25-5.35 \mathrm{GHz}$, and 5.725-5.825 GHz. They belong to the $\mathrm{C}$ band of radar which is usually adopted by remote control and guide radar. Here, we assume that the center frequency $f_{c}$ of the IRCS is $5.8 \mathrm{GHz}$, the number of antennas is $M=4$, the maximum relative velocity between the IRCS and targets is $v_{\max , r}=800 \mathrm{~m} / \mathrm{s}$, i.e., the maximum relative velocity between the IRCS and communication receiver is $v_{\max , c}=1600 \mathrm{~m} / \mathrm{s}$, the demands for the MUR is $150 \mathrm{~km}$, range resolution is $15 \mathrm{~m}$, and blind zone is less than $15 \mathrm{~km}$.

The $v_{\max }=800 \mathrm{~m} / \mathrm{s}$ corresponds to the Doppler shift of $30.93 \mathrm{kHz}$, so the subcarrier spacing is desired to guarantee $\Delta f \geqslant 309.3 \mathrm{kHz}$. Furthermore, physical layer convergence protocol in 802.11a suggests that the IFFT/FFT period is $T=3.2 \mu \mathrm{s}$ in accordance with $\Delta f=312.5$ $\mathrm{kHz}$, and the cyclic prefix is $0.8 \mu \mathrm{s}$. Hence, we choose the subcarrier spacing of $\Delta f=312.5 \mathrm{kHz}$. According to the MUR of $150 \mathrm{~km}$, blind zone of less than $15 \mathrm{~km}$, and the range resolution of $15 \mathrm{~m}$ we can obtain that the PRF is $f_{r}=1 \mathrm{kHz}$, the system bandwidth is $B_{s} 10 \mathrm{MHz}$, and the 
pulse duration is $T_{p} \leqslant 100 \mu$ s. Substituting $f_{r}=1 \mathrm{kHz}$ and $f_{c}=5.8 \mathrm{GHz}$ into (2) yields the MUV $v_{u}=25.86$ $\mathrm{m} / \mathrm{s}$, which cannot suffice the requirement of velocity detection. Thus, some resolving velocity ambiguity methods are required to be employed. Since $B_{s}=M N_{c} \Delta f$ is 10 $\mathrm{MHz}$ and $\Delta f=312.5 \mathrm{kHz}$, the $N_{c}=8$ is obtained. Since $T_{p}=N_{s}\left(T_{g}+\frac{1}{\Delta f}\right)$, the $N_{s} \leqslant 25$. The object function implies that the greater the $N_{s}$, the larger the channel capacity. Hence, we choose $N_{s}=25$. If the communication uses quadrature phase shift keying (QPSK) modulation, the communication data rate $R_{b}$ will be $2.4 \mathrm{Mbit} / \mathrm{s}$.

The final IRCS parameters and performance indexes are summarized in Case 1 of Table 1. In Table 1, we also give the other two quintessential cases. Case 2 has less blind zone with lower data rate and Case 3 has larger MUV with smaller MUR. The performance and IRCS parameters of Case 2 and Case 3 are similarly confirmed as Case 1 .

Table 1 IRCS parameters and performance indexes

\begin{tabular}{ccccc}
\hline Symbol & Parameter & Case 1 & Case 2 & Case 3 \\
\hline$f_{c} / \mathrm{GHz}$ & Center frequency & 5.8 & 5.8 & 5.8 \\
$M$ & Number of elements & 4 & 4 & 4 \\
$N_{s}$ & Number of OFDM symbols & 25 & 2 & 2 \\
$N_{c}$ & Number of carrier & 8 & 8 & 8 \\
$R_{b} /(\mathrm{Mbit} / \mathrm{s})$ & Data rate (QPSK) & 1.6 & 0.128 & 1.28 \\
$T / \mu \mathrm{s}$ & Elementary symbol duration & 3.2 & 3.2 & 3.2 \\
$T_{g} / \mu \mathrm{s}$ & Length of CP & 0.8 & 0.8 & 0.8 \\
$f_{r} / \mathrm{kHz}$ & PRF & 1 & 1 & 10 \\
$R_{u} / \mathrm{km}$ & MUR & 150 & 150 & 15 \\
$R_{b z} / \mathrm{km}$ & Blind zone & 15 & 1.2 & 1.2 \\
$\Delta R / \mathrm{m}$ & Range resolution & 15 & 15 & 15 \\
$v_{u} /(\mathrm{m} / \mathrm{s})$ & MUV & 25.86 & 25.86 & 258.62 \\
\hline
\end{tabular}

\subsection{IRCS performance}

In this section, several numerical examples are presented to illustrate the performance of IRCS.

In Fig. 10, we plot the variation of data rate and blind zone with the number of symbols. In the simulation, four antennas with half wavelength interval transmit different OFDM signals which consist of eight subcarriers with the subcarrier interval $312.5 \mathrm{kHz}$ and the length of $\mathrm{CP} 0.8 \mu \mathrm{s}$. Moreover, the PRF is $1 \mathrm{kHz}$, and the number of OFDM symbols with QPSK modulation in each pulse varies from 2 to 22 stepping by 2 . The data rate of real IRCS is indicated by "Real", and the AWGN Shannon capacity with SNR $10 \mathrm{~dB}$ is represented by "Shannon". We can see that with the increasing of the number of OFDM symbols in each pulse (i.e., the pulse duration is increased), the communication data rate is improved. However, the blind zone of radar is enlarged, which is unfavourable for radar detection. Hence, it is imperative to make a trade off in the number of OFDM symbols in each pulse.

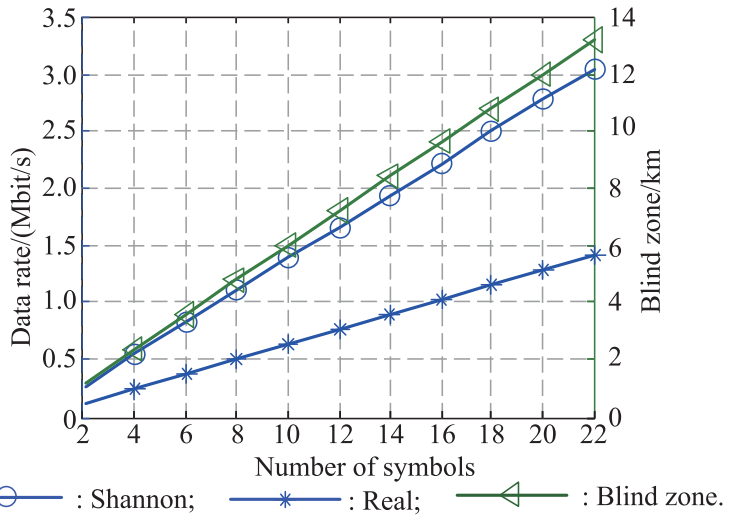

Fig. 10 Variation of data rate and blind zone with the number of symbols

The dependence of data rate and angle resolution upon the number of antennas is depicted in Fig. 11. Each pulse contains five OFDM symbols and the number of antennas ranges from 2 to 20 stepping by 2 . The other simulation conditions are the same as those in Fig. 10. The proposed IRCS and conventional phased array are represented by "IRCS" and "Phased array", respectively. It is obvious that the communication data rate and angle resolution are enhanced with the number of antennas increased. Furthermore, the angle resolution of IRCS is higher than that of the phased array with the same number of antennas. The reason is that the array aperture of IRCS is doubled through utilizing the transmitted degrees of freedom. These results lead us to draw a conclusion that the more the antennas is, the better the radar and communication performance of IRCS will be if the system size and energy cost can support.

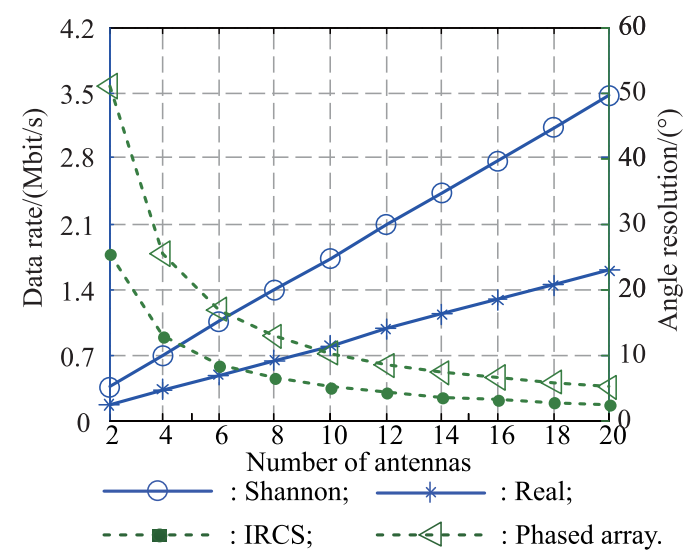

Fig. 11 Variation of data rate and angle resolution with the number of antennas

The simulation results of the data rate, MUR and MUV as functions of PRF are shown in Fig. 12 and Fig. 13. In the simulations, the PRF varies from $0.2 \mathrm{kHz}$ to $9.8 \mathrm{kHz}$ stepping by $0.4 \mathrm{kHz}$, center frequency is $5.8 \mathrm{GHz}$, and 
the other conditions are consistent with those in Fig. 10. We can see that the data rate of communication and MUV are increasing with the enhancement of PRF whereas the MUR is decreasing. From these results, it can be concluded that the increase of PRF is beneficial to communication; in contrast, it is disadvantageous as well as advantageous to radar. Therefore, a compromise should be made in the design of PRF.

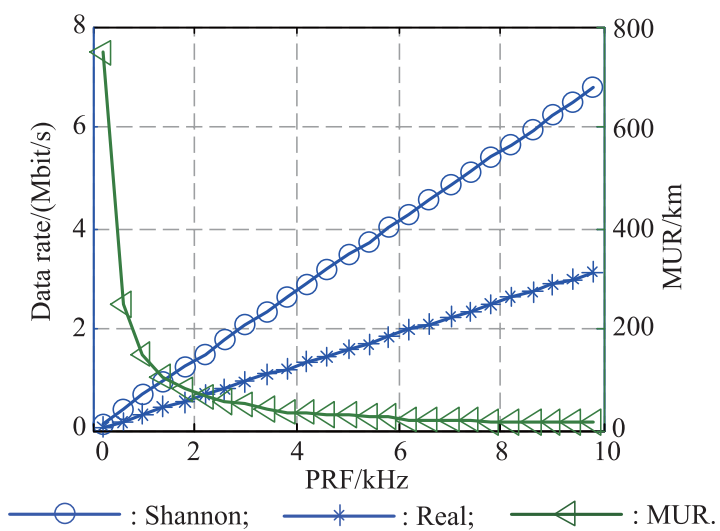

Fig. 12 Variation of data rate and MUR with PRF

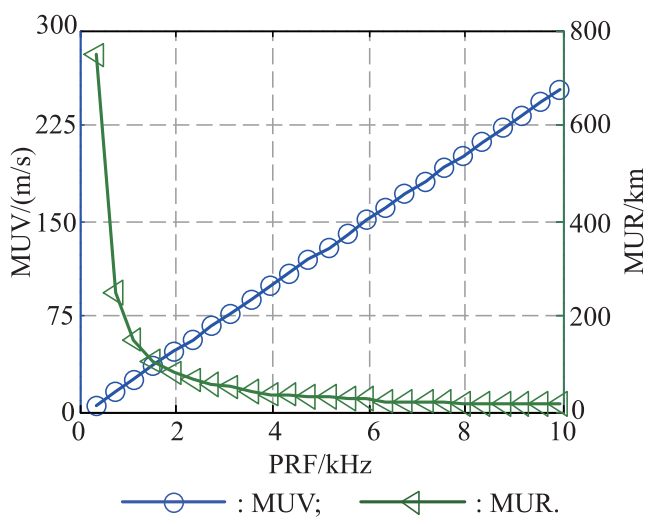

Fig. 13 Variation of MUR and MUV with PRF

In Fig. 14, the variation of data rate with MUR is plotted. The IRCS in [36] is denoted as "S-IRCS". In the simulation, for the IRCS the number of subcarriers employed by each antenna is 64 , the blind zone is maintained to be 1.56 $\mathrm{km}$. The other simulation conditions are similar to those in Fig. 11. With the increasing of MUR, the data rate is decreasing, and the duty ratio for IRCS is also decreasing due to the increase of PRI. Moreover, for the same MUR, the data rate of IRCS is more excellent than the S-IRCS, since the S-IRCS transmits only a part of subcarriers to improve the MUR.

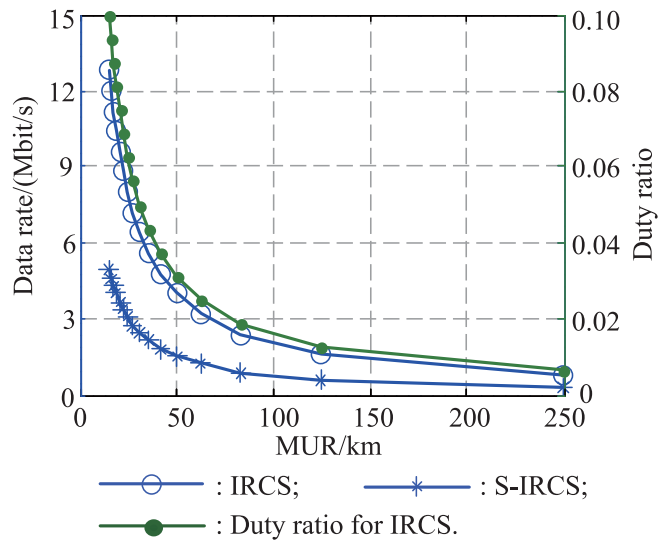

Fig. 14 Variation of data rate with MUR

The comparison of angle resolution is illustrated in Fig. 15. In the simulation, the number of antennas is 10 , and other simulation conditions are the same as those in Fig. 10. Since the angle resolution of S-IRCS is the same as IRCS, the results of IRCS are only plotted. For the same antenna configuration, the angle resolution of the IRCS is the same as the traditional MIMO radar but higher than the conventional phased array, since the IRCS has the property of MIMO radar that the virtual aperture is improved two times.

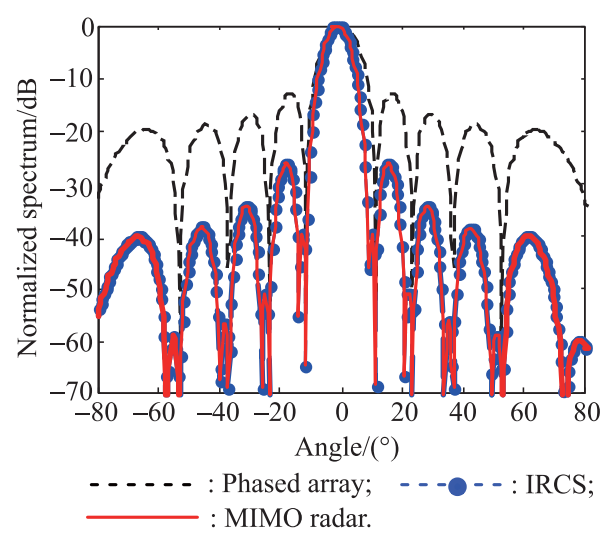

Fig. 15 Comparison of angle resolution

In Fig. 16, the bit error rate (BER) of OFDM in Gaussian channel with respect to SNR under different modulation styles or data rates is plotted. In the simulation, each pulse contains 25 OFDM symbols with 16 subcarriers and the other conditions are in line with those in Fig. 10. It is evident that the BER is enhanced with the improvement of the data rate. This is due to the fact that coding at higher rate generates lower bit energy to noise power spectral density ratio. That is to say we can improve the data rate by enhancing the coding rate with the decrease of communication reliability. 


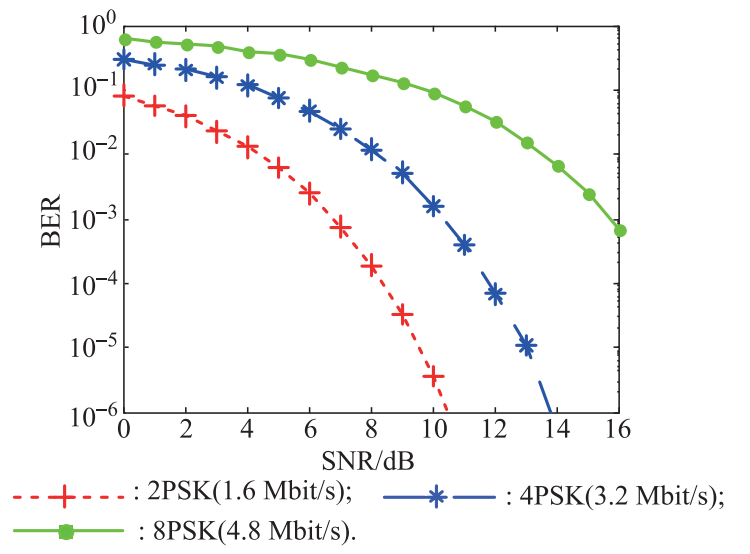

Fig. 16 Comparison of BER with respect to SNR under different data rates

\section{Conclusions}

In this paper, a new integrated radar and communication system combining the MIMO system with OFDM waveform is proposed. The underlying performance improvement in angle resolution and data rate is investigated. The feasibility of the proposed system and different requirements of radar and communication are discussed. According to these requirements, an optimization problem is established to obtain suitable system parameters. In three typical cases, the system parameters that satisfy both the radar and communication limitations are derived. Moreover, the dependence of radar and communication performance indexes on the system parameters is illustrated. Compared with the SISO system, the proposed system can improve the angle resolution, and data rate.

According to the simulation results, the communication performance of the proposed IRCS is improved with the increasing of SNR, the number of antennas, PRF and the number of symbols in each pulse. The increase of the number of antennas improves the angle resolution, whereas both the PRF and the number of symbols have a contradictory influence on the radar performance, which compels us to make a tradeoff. Moreover, the proposed system is applicable for long range surveillance, and has higher data rate, simultaneously.

For the MIMO-OFDM IRCS, some specific hardware actualization and radar signal processing problems are deserved future research, and now we are devoting to the corresponding radar signal processing.

\section{References}

[1] G. Tavik, C. Hilterbrick, J. Evins, et al. The advanced multifunction RF concept. IEEE Trans. on Microwave Theory and Techniques, 2005, 53(3): 1009-1020.

[2] L. Han, K. Wu. Multifunctional transceiver for future intelligent transportation systems. IEEE Trans. on Microwave Theory and Techniques, 2011, 59(7): 1879-1892.
[3] L. Han, K. Wu. 24-GHz integrated radio and radar system capable of time-agile wireless communication and sensing. IEEE Trans. on Microwave Theory and Techniques, 2012, 60(3): $619-631$.

[4] C. Sturm, W. Wiesbeck. Waveform design and signal processing aspects for fusion of wireless communications and radar sensing. IEEE Trans. on Proceedings, 2011, 99(7): 12361259.

[5] A. Hassanien, M. G. Amin, Y. D. Zhang, et al. Dual-function radar-communications: information embedding using sidelobe control and waveform diversity. IEEE Trans. on Signal Processing, 2016, 64(8): 2168-2181.

[6] H. Takahara, K. Ohno, M. Itami. A study on UWB radar assisted by inter-vehicle communication for safety applications. Proc. of the IEEE International Conference on Vehicular Electronics and Safety, 2012: 99-104.

[7] A. K. Mishra, M. Inggs. FOPEN capabilities of commensal radars based on whitespace communication systems. Proc. of the IEEE Conference on Electronics, Computing and Communication Technologies, 2014: 1-5.

[8] H. Takase, M. Shinriki. A dual-use radar and communication system with complete complementary codes. Proc. of the 15th International Radar Symposium, 2014: 16-18.

[9] R. M. Mealey. A method for calculating error probabilities in a radar communication system. IEEE Trans. on Space Electronics and Telemetry, 1963, 9(2): 37-42.

[10] D. Ciuonzo, A. D. Maio, G. Foglia, et al. Intrapulse radarembedded communications via multiobjective optimization. IEEE Trans. on Aerospace and Electronic Systems, 2015, 51(4): 2960-2974.

[11] Y. L. Sit, T. Zwick. MIMO OFDM radar with communication and interference cancellation features. Proc. of IEEE Radar Conference, 2014: 19-23.

[12] Y. L. Sit, L. Reichardt, C. Sturm, et al. Extension of the OFDM joint radar-communication system for a multipath, multiuser scenario. Proc. of IEEE Radar Conference, 2011: 718-723.

[13] J. J. Zhao, K. Huo, X. Li. A chaos-based phase-coded OFDM signal for joint radar-communication systems. Proc. of the 12th International Conference on Signal Processing, 2014: 1997 2002.

[14] L. Hu, Z. C. Du, G. R. Xue. Radar-communication integration based on OFDM signal. Proc. of the IEEE International Conference on Signal Processing, Communications and Computing, 2014: $442-445$.

[15] D. Garmatyuk, K. Kauffman, J. Schuerger, et al. Wideband OFDM system for radar and communications. Proc. of IEEE Radar Conference, 2009: 1-6.

[16] IEEE Std 802.11a-1999, Part 11: Wireless LAN medium access control (MAC) and physical layer (PHY) specifications: high-speed physical layer in the $5 \mathrm{GHz}$ band.

[17] R. F. Tigrek, W. J. A. de Heij, P. van Genderen. Multi-carrier radar waveform schemes for range and Doppler processing. Proc. of the IEEE Radar Conference, 2009: 1-5.

[18] S. Sen, A. Nehorai. Sparsity-based multi-target tracking using OFDM radar. IEEE Trans. on Signal Processing, 2011, 59(4): $1902-1906$.

[19] S. Sen. OFDM radar space-time adaptive processing by exploiting spatio-temporal sparsity. IEEE Trans. on Signal Processing, 2013, 61(1): 118-130.

[20] T. X. Zhang, X. G. Xia. OFDM synthetic aperture radar imaging with sufficient cyclic prefix. IEEE Trans. on Geoscience and Remote Sensing, 2015, 53(1): $394-404$.

[21] S. Sen, A. Nehorai. Adaptive OFDM radar for target detection in multipath scenarios. IEEE Trans. on Signal Processing, 2011, 59(1): 78-90.

[22] H. Bölcskei, D. Gesbert, A. J. Paulraj. On the capacity of OFDM-based spatial multiplexing systems. IEEE Trans. on 
Communications, 2002, 50(2): 225-234.

[23] J. G. Proakis, M. Salehi. Digital communications. 5th edition. New York: McGraw-Hill Science, 2007.

[24] E. Fishler, A. Haimovich, R. Blum, et al. MIMO radar: an idea whose time has come. Proc. of the IEEE Radar Conference, 2004: $71-78$.

[25] B. J. Donnet, I. D. Longstaff. MIMO radar, techniques and opportunities. Proc. of the 3rd European Radar Conference, 2006: $112-115$.

[26] J. Li, P. Stoica. MIMO radar signal processing. New Jersey: John Wiley and Sons Inc., 2009.

[27] H. Bölcskei, M. Borgmann, A. J. Paulraj. Impact of propagation environment on the performance of space-frequency coded MIMO-OFDM. IEEE Journal on Selected Areas in Communications, 2003, 21(3): 427-439.

[28] K. W. Park, Y. S. Cho. An MIMO-OFDM technique for highspeed mobile channels. IEEE Communications Letters, 2005, 9(7): $604-606$.

[29] Y. H. Cao, X. G. Xia, S. H. Wang. IRCI free colocated MIMO radar based on sufficient cyclic prefix OFDM waveforms. IEEE Trans. on Aerospace and Electronic Systems, 2015, 51(3): $2107-2120$.

[30] Y. H. Cao, X. G. Xia. IRCI free MIMO-OFDM SAR using circularly shifted Zadoff-Chu sequences. IEEE Geoscience and Remote Sensing Letters, 2015, 12(5): 1126-1130.

[31] X. G. Xia, T. X. Zhang, L. J. Kong. MIMO OFDM radar IRCI free range reconstruction with sufficient cyclic prefix. IEEE Trans. on Aerospace and Electronic Systems, 2015, 51(3): $2276-2293$.

[32] X. H. Wu, A. A. Kishk, A. W. Glisson. MIMO-OFDM radar for direction estimation. IET Radar Sonar and Navigation, 2010, 4(1): $28-36$.

[33] S. Sen, A. Nehorai. OFDM MIMO radar design for low-angle tracking using mutual information. Proc. of the 3rd IEEE International Workshop on Computational Advances in MultiSensor Adaptive Processing, 2009: 173-176.

[34] S. Sen, A. Nehorai. OFDM MIMO radar with mutualinformation waveform design for low-grazing angle tracking. IEEE Trans. on Signal Processing, 2010, 58(6): 3152 - 3162.

[35] C. Sturm, Y. L. Sit, M. Braun, et al. Spectrally interleaved multi-carrier signals for radar network applications and multiinput multi-output radar. IET Radar Sonar Navigation, 2013, 7(3): $261-269$

[36] B. J. Donnet, I. D. Longstaff. Combining MIMO radar with OFDM communications. Proc. of the 3rd European Radar Conference, 2006: 13-15.

[37] Y. J. Kim, X. Li, N. Y. Park. Generalized suboptimum decoding for STTC-OFDM systems in MIMO Rayleigh fading channels. International Symposium on Communications and Information Technologies, 2007: 194-197.

[38] H. Y. Chen, J. N. Lin, H. S. Hu, et al. STBC-OFDM downlink baseband receiver for mobile WMAN. IEEE Trans. on Very Large Scale Integration Systems, 2013, 21(1): 43-54.

[39] Y. Chanho, L. Hoojin, K. Joonhyuk. Combined ML and DFE decoding for coded double STBC-OFDM system. IEEE Communications Letters, 2010, 14(12): 1164-1166.

[40] H. Dogan, H. A. Cirpan, E. Panayirci. Iterative channel estimation and decoding of Turbo coded SFBC-OFDM systems. IEEE Trans. on Wireless Communications, 2007, 6(8): 3090 3101.
[41] S. Boyd, L. Vandenberghe. Convex optimization. New York: Cambridge University Press, 2004.

[42] M. Gen, R. Cheng. Genetic algorithms and engineering optimization. New Jersey: John Wiley \& Sons, 2000.

\section{Biographies}

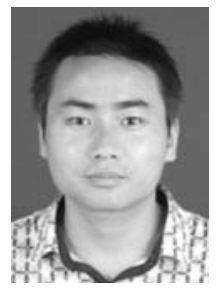

Yongjun Liu was born in 1990. He received his B.S. degree in electronic and information engineering from Xidian University, Xi'an, China, in 2013. He is currently working toward his Ph.D. degree in the National Laboratory of Radar Signal Processing, Xidian University. His research interests include array radar signal processing and integrated waveform design.

E-mail: yjliuinsist@163.com

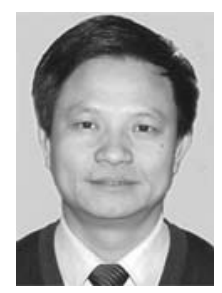

Guisheng Liao was born in 1963. He received his B.S. degree from Guangxi University, Guangxi, China, and M.S. and Ph.D. degrees from Xidian University, Xi' an, China, in 1985, 1990, and 1992, respectively. $\mathrm{He}$ is currently a professor at the $\mathrm{Na}-$ tional Laboratory of Radar Signal Processing, Xidian University. He has been a senior visiting scholar in the Chinese University of Hong Kong, Hong Kong. His research interests include array signal processing, space-time adaptive processing, SAR ground moving target indication, and distributed small satellite SAR system design. Dr. Liao is a member of the National Outstanding Person and the Cheung Kong Scholars in China.

E-mail: liaogs@xidian.edu.cn

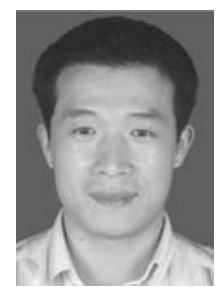

Zhiwei Yang was born in 1980 . He received his M.S. and Ph.D. degrees in electrical engineering from Xidian University, Xi' an, China, in 2005 and 2008 respectively. He is currently an associate professor with the National Laboratory of Radar Signal Processing, Xidian University. His current work includes adaptive array signal processing, space-timepolarmetric adaptive processing and he designed the ground moving target indication algorithms for the space-borne SAR/GMTI systems in China.

E-mail: yangzw@xidian.edu.cn

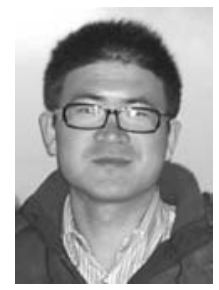

Jingwei Xu was born in 1987. He received his B.S. degree in electronic and information engineering, and Ph.D. degree in signal and information processing, both from Xidian University, China, in 2010 and 2015 , respectively. He is currently a postdoctoral researcher in the National Laboratory of Radar Signal Processing, Xidian University. His research interests include robust adaptive beamforming, frequency diverse array radar systems, STAP-based radar signal processing, and MIMO radar signal processing.

E-mail: xujingwei1987@163.com 